\title{
First Assessment of ENDF/B-VIII and EPICS Atomic Data Libraries
}

\author{
Min Cheol Han, Maria Grazia Pia ${ }^{\circledR}$, Paolo Saracco ${ }^{(}$, and Tullio Basaglia
}

\begin{abstract}
This paper reports an extensive assessment of widely used evaluated atomic data libraries released in ENDF/B-VIII.0 and in EPICS2017 in early 2018. The new versions are intended to replace the data libraries currently used by major Monte Carlo particle transport codes to model electron and photon interactions with matter, which date back to the 1990s. The evaluation is performed from a user perspective and concerns various characteristics of the data, including their intrinsic consistency, the differences across their various formats and distribution sources, and the effects on computational performance associated with their use. The results of the tests demonstrate the impact of using the new data libraries in a Monte Carlo simulation environment and highlight some opportunities for improvement in future versions.
\end{abstract}

Index Terms-Cross sections, Monte Carlo, quality assessment, radiation transport modeling, simulation.

\section{INTRODUCTION}

$\mathbf{E}$ VALUATED data libraries are an essential instrument in Monte Carlo particle transport and in various physics and engineering applications, where reliable modeling and fast computation of particle interactions with matter are required. They consist of tabulations of relevant physics quantities, such as cross sections, secondary particle spectra, nuclear and atomic parameters, which derive from the evaluation of the available body of knowledge of theoretical computations, experimental measurements, or both, to distill reliable references for computational physics applications.

Among the data libraries most commonly used in nuclear applications are BROND (Russian Evaluated Neutron Data Library) [1], CENDL (Chinese Evaluated Nuclear Data Library) [2], ENDF/B (Evaluated Nuclear Data File) [3], JEFF (Joint Evaluated Fission and Fusion File) [4] and JENDL (Japanese Evaluated Nuclear Data Library) [5]. Due to the complexity of the evaluation process, major releases of these data libraries, which encompass significant evolutions in their content, are not frequent; for instance, the last major releases of ENDF/B occurred in 1990 (ENDF/B-VI [6]), 2006 (ENDF/B-VII [7]) and 2018 (ENDF/B-VIII [3]).

Manuscript received March 28, 2018; revised June 7, 2018; accepted June 17, 2018. Date of publication June 20, 2018; date of current version August 15, 2018.

M. C. Han, M. G. Pia, and P. Saracco are with the INFN Sezione di Genova, I-16146 Genoa, Italy (e-mail: mincheol.han@ge.infn.it; mariagrazia.pia@ge.infn.it; paolo.saracco@ge.infn.it).

T. Basaglia is with CERN, CH-1211 Geneva, Switzerland (e-mail: tullio.basaglia@cern.ch).

Color versions of one or more of the figures in this paper are available online at http://ieeexplore.ieee.org.

Digital Object Identifier 10.1109/TNS.2018.2849328
From a user perspective, a careful assessment of the impact of changes and of new features is needed before a new version of the data libraries is adopted for production in an experimental application environment. This paper documents an extensive assessment of content features and computational performance of the atomic data libraries included in ENDF/B-VIII, released on February 2, 2018. It also shows their relationship with the release of the same libraries by IAEA as EPICS2017 [9]-[11] in January 2018, and appraises the evolution of the data with respect to the previous versions, which are currently used by several general purpose Monte Carlo transport codes. Given the short lapse of time since the release of ENDF/B-VIII and EPICS2017, to the best of our knowledge, this paper documents the first assessment, from a user perspective, of the new atomic data libraries available to the experimental community.

This evaluation is intended to document some relevant issues concerning the use of the new version of these data libraries in Monte Carlo simulation applications and to provide helpful feedback for their future improvement. The verification process conforms to [8]. Due to its intrinsic complexity, the validation of the physics content of these data libraries is documented in detail in dedicated papers.

\section{DATA LiBRARIES FOR ELECTRON-PHOTON TRANSPORT}

A set of evaluated data libraries relevant to electronphoton transport in matter was created by the Lawrence Livermore National Laboratory (LLNL). This collection, originally known as the electromagnetic component of the "Livermore Library," encompasses three libraries: the Evaluated Atomic Data Library (EADL) [12], containing atomic parameters, the Evaluated Electron Data Library (EEDL) [13] and the Evaluated Photon Data Library (EPDL) [14], containing cross section data and related physical quantities pertinent to electron and photon interactions with atoms. The International Atomic Energy Agency (IAEA) has taken over their distribution since 2014, releasing the collection under the name of Electron Photon Interaction Cross Sections (EPICS) [15].

The three libraries are available in two formats: ENDF-6 [16] and ENDL [17]. The data components differ across the two formats; with few exceptions, the libraries released in ENDL format contain a larger number of physics components. The content of the three data libraries available in either format is summarized in Tables I-III; as documentation of the physics data available in either format is not easily 
TABLE I

EPDL CONTENT

\begin{tabular}{|c|c|c|c|c|c|c|}
\hline \multirow{2}{*}{ Physics Data } & \multicolumn{2}{|c|}{ EPDL97 } & \multicolumn{2}{|c|}{ EPICS2014 } & \multicolumn{2}{|c|}{ EPICS2017 } \\
\hline & ENDL & ENDF-6 & ENDL & ENDF-6 & ENDL & ENDF-6 \\
\hline Total photon cross section & - & - & - & - & - & yes \\
\hline Coherent scattering: integrated cross section & yes & yes & yes & yes & yes & yes \\
\hline Coherent scattering: average energy of the scatterd photon & yes & - & yes & - & yes & - \\
\hline Coherent scattering: form factor & yes & yes & yes & yes & yes & yes \\
\hline Coherent scattering: imaginary anomalous scattering factor & yes & yes & yes & yes & yes & yes \\
\hline Coherent scattering: real anomalous scattering factor & yes & yes & yes & yes & yes & yes \\
\hline Incoherent scattering: integrated cross section & yes & yes & yes & yes & yes & yes \\
\hline Incoherent scattering: scattering function & yes & yes & yes & yes & yes & yes \\
\hline Incoherent scattering: average energy of the secondary particles & yes & - & yes & - & yes & - \\
\hline Photoelectric: integrated cross section & yes & yes & yes & yes & yes & yes \\
\hline Photoelectric: average energy to the residual atom & yes & - & yes & - & - & - \\
\hline Photoelectric: average energy of secondary particles & yes & - & yes & - & - & - \\
\hline Photoelectric: cross section by subshell & yes & yes & yes & yes & yes & yes \\
\hline Photoelectric: average energy to the residual atom by subshell & yes & - & yes & - & yes & - \\
\hline Photoelectric: average energy of secondary particles by subshell & yes & - & yes & - & yes & - \\
\hline Pair production: integrated cross section & yes & yes & yes & yes & yes & yes \\
\hline Pair production: average energy of secondary particles & yes & - & yes & - & yes & - \\
\hline Triplet production: integrated cross section & yes & yes & yes & yes & yes & yes \\
\hline Triplet production: average energy of secondary particles & yes & - & yes & - & yes & - \\
\hline Pair and triplet production: integrated cross section & - & yes & - & yes & - & yes \\
\hline
\end{tabular}

TABLE II

EADL CONTENT

\begin{tabular}{|c|c|c|c|c|c|c|}
\hline \multirow{2}{*}{ Physics Data } & \multicolumn{2}{|c|}{ EADL91 } & \multicolumn{2}{|c|}{ EPICS2014 } & \multicolumn{2}{|c|}{ EPICS2017 } \\
\hline & ENDL & ENDF-6 & ENDL & ENDF-6 & ENDL & ENDF-6 \\
\hline Number of electrons & yes & yes & yes & yes & yes & yes \\
\hline Binding energy & yes & yes & yes & yes & yes & yes \\
\hline Kinetic energy & yes & - & yes & - & yes & - \\
\hline Average radius & yes & - & yes & - & yes & - \\
\hline Radiative level width & yes & - & yes & - & yes & - \\
\hline Non-radiative level width & yes & - & yes & - & yes & - \\
\hline Average energy to the residual atom per initial vacancy & yes & - & yes & - & yes & - \\
\hline Average energy of particles per initial vacancy & yes & - & yes & - & yes & - \\
\hline Average number of particles per initial vacancy & yes & - & yes & - & yes & - \\
\hline Radiative transition probability and emitted particle energy & yes & yes & yes & yes & yes & yes \\
\hline Non-radiative transition probability and emitted particle energy & yes & yes & yes & yes & yes & yes \\
\hline
\end{tabular}

retrievable, it was identified by parsing the respective data files.

Along with their distribution as standalone data libraries, EPDL, EEDL, and EADL in ENDF-6 format are also included in the ENDF/B nuclear data system, where they are identified as photo-atomic, electro-atomic, and atomic relaxation libraries, respectively.

The EADL, EEDL, and EPDL data libraries are widely used by general-purpose Monte Carlo particle transport codes, including EGSnrc [18], FLUKA [19], [20], Geant4 [21]-[23], MCNP [24], and Penelope [25].

\section{A. "Livermore" Data Libraries: EADL, EEDL, and EPDL}

The three data libraries are intended to be mutually compatible to facilitate their use in the electron-photon transport: they cover the same elements, with atomic number from 1 to 100 , and the same energy range, from $10 \mathrm{eV}$ to $100 \mathrm{GeV}$; they are based on the same atomic parameters, e.g. they were created using the same subshell electron binding energies.

The EPDL data library underwent several updates [14], [26]-[30]; the latest version EPDL97, released by LLNL in 1997, encompasses data to describe photon coherent and incoherent scattering, the photoelectric effect, and pair and triplet production. EPDL97 also incorporates the Evaluated Excitation Data Library (EXDL), which includes data to describe photoexcitation lines. Although EXDL is distributed with EPDL97, it is configured as a separate file. An overview of the EPDL physics content is summarized in Table I; further details can be found in the previously cited references.

The EADL library was first released in 1991. It contains data for isolated neutral atoms: subshell parameters, relaxation parameters, and energy deposition terms. The parameters listed for each subshell are the number of electrons, the electron binding energy and kinetic energy, and the expectation value of the radius. Relaxation data concern both the radiative and nonradiative transitions. Fluorescence yields are derived from transition probabilities, and energy deposition terms are estimated taking into account transition probabilities and binding energies.

The atomic subshell parameters collected in EADL are based on theoretical calculations by Scofield [31]-[33] and Chen et al. [34]-[37]; in addition to published references, 
TABLE III

EEDL CONTENT

\begin{tabular}{|c|c|c|c|c|c|c|}
\hline \multirow{2}{*}{ Physics Data } & \multicolumn{2}{|c|}{ EEDL91 } & \multicolumn{2}{|c|}{ EPICS2014 } & \multicolumn{2}{|c|}{ EPICS2017 } \\
\hline & ENDL & ENDF-6 & ENDL & ENDF-6 & ENDL & ENDF- 6 \\
\hline Total electron cross section & - & - & - & - & - & yes \\
\hline Large angle elastic scattering: integrated cross section & yes & yes & yes & yes & yes & yes \\
\hline Large angle elastic scattering: average energy to the residual atom & yes & - & yes & - & yes & - \\
\hline Large angle elastic scattering: average energy of the scattered electron & yes & - & yes & - & yes & - \\
\hline Large angle elastic scattering: angular distributions & yes & yes & yes & yes & yes & yes \\
\hline Elastic scattering: integrated cross section & yes & - & yes & - & yes & yes \\
\hline Ionisation: integrated cross section & - & - & - & - & yes & yes \\
\hline Ionisation cross section by subshell & yes & yes & yes & yes & yes & yes \\
\hline Ionisation: average energy of secondary particles by subshell & yes & - & yes & - & yes & - \\
\hline Ionisation: spectra of the recoil electron by subshell & yes & yes & yes & yes & yes & yes \\
\hline Bremsstrahlung: integrated cross section & yes & yes & yes & yes & yes & yes \\
\hline Bremsstrahlung: energy spectra of the secondary photon & yes & yes & yes & yes & yes & yes \\
\hline Bremsstrahlung: average energy of the secondary photon & yes & yes & yes & yes & yes & yes \\
\hline Bremsstrahlung: average energy of the secondary electron & yes & - & yes & - & yes & - \\
\hline Excitation: integrated cross section & yes & yes & yes & yes & yes & yes \\
\hline Excitation: average energy to the residual atom & yes & yes & yes & yes & yes & yes \\
\hline
\end{tabular}

EADL documentation cites "private communications," which hinder the full identification of the origin of the tabulated data.

The physical content of EADL is summarized in Table II; one can notice that the library in ENDF-6 format includes only a small subset of the data available in the ENDL format.

The EEDL library was originally released by LLNL in 1991; it covers the energy range from $5 \mathrm{eV}$ to $100 \mathrm{GeV}$. The cross sections tabulated in EEDL are based on Seltzer's calculation method [38], which distinguishes close and distant collisions. The atomic parameters required in these calculations were taken from EADL [12], while the subshell photoelectric cross sections required for the distant-collision component were derived from the EPDL version of 1989 [30]. An overview of the EEDL physics content is summarized in Table III.

The documentation associated with EPDL and EEDL recommends using logarithmic interpolation to calculate physics quantities based on the tabulated values, with the only exception of anomalous scattering factors, which should be linearly interpolated due to the fact that the data can be negative.

For convenience, the latest versions of libraries released by LLNL are identified in the following sections as EADL91, EEDL91, and EPDL97, or generically as "original Livermore libraries."

\section{B. EPICS}

Two versions, EPICS2014 and EPICS2017, released in September 2014 and in January 2018, respectively, are available at the time of writing this paper. The IAEA also distributes the 1991 version of EADL and EEDL, and the 1997 version of EPDL that make up the original "Livermore Library."

EPICS2014 is also released by the Nuclear Energy Agency (NEA) as ZZ-EPICS2014 with package identifier IAEA1435/01 and by the Radiation Safety Information Computational Center (RSICC) as Code Package DLC-272; however, the versions distributed by NEA and RSICC do not appear up to date compared with the version that can be downloaded from the IAEA, which incorporates corrections released at a later date.

EPICS2017 is also distributed by the National Nuclear Data Center (NNDC) [39]. The version available from NNDC differs from the version distributed by IAEA.

The mention of EPICS, EPICS2014, and EPICS2017 in this paper refers to the data libraries released by IAEA, unless other release sources are explicitly specified.

The documentation of EPICS2014 [40] indicates that the data libraries have been "modernized" by recently published data, but it does not specify which physics data have been updated nor the references from which more modern data have been taken. An assessment of the evolution of the physics content of EPICS2014 is documented in Section IV. In addition, tabulated numbers have been modified for consistency with scientific data notation.

A previous study of several atomic binding energy compilations [41] highlighted various issues related to the values included in EADL. The EPICS2017 documentation [9]-[11] reports that new atomic binding energies have been adopted in the data libraries and related physical quantities, such as cross sections and secondary particle features, have been updated for data consistency.

Total electron and photon interaction cross sections, as well as integrated ionization cross sections, are first included in EPICS2017 [11], while the data set representing the average energy of the secondary particles for photoelectric effect is no longer distributed. Large angle elastic scattering and its angular distribution have been added in the ENDF- 6 format of EPICS2017 [11].

In addition, according to the documentation [9], [10], the number of points tabulated in the physics data sets of the new libraries has been increased by approximately a factor three to enable linear interpolation instead of previously recommended logarithmic interpolation.

A few format conversion errors present in previously released data libraries have been corrected and documented in EPICS: a conversion error between the ENDL and ENDF-6 
TABLE IV

Sources of ATOMIC Data Libraries USED IN ENDF/B

\begin{tabular}{lccc}
\hline Version of ENDF/B & $\begin{array}{c}\text { Atomic } \\
\text { relaxation } \\
\text { data }\end{array}$ & $\begin{array}{c}\text { Electron } \\
\text { interaction } \\
\text { data }\end{array}$ & $\begin{array}{c}\text { Photon } \\
\text { interaction } \\
\text { data }\end{array}$ \\
\hline VIII.0 & EPICS2017 & EPICS2017 & EPICS2017 \\
VIII.beta0 - beta7 & EPICS2014 & EPICS2014 & EPICS2014 \\
VII.1 & EADL91 & EEDL91 & EPDL97 \\
VI.8 & EADL91 & EADL91 & EPDL97 \\
VI.0 & & & EPDL89 \\
\hline
\end{tabular}

formats, concerning the average energy loss by an electron due to Bremsstrahlung in EEDL91, was corrected in EPICS2014 [40]; a mistake concerning the total electron scatter cross section in ENDF-6 format, which erroneously reported large angle scatter data in EEDL91 and EPICS2014, has been corrected in EPICS2017 [11].

The nominal content of EPICS2014 and 2017 is compared to that of the original Livermore Library in Tables I-III. An assessment of the differences observed between EPICS2014, EPICS2017, and the original EADL91, EEDL91, and EPDL97 is documented in Section IV.

\section{ENDF/B Atomic Data Libraries}

The ENDF/B data library currently encompasses the content of EADL, EEDL and EEDL (limited to the data sets listed in Tables I-III that are available in an ENDF-6 format). All three data libraries have been incorporated since version VI.8; previous versions included only EPDL.

The original versions released by LLNL, i.e. EADL91, EEDL91 and EPDL97, have been available in ENDF/B versions VI.8 through VII.1; the libraries belonging to EPICS2014 were introduced in ENDF/B-VIII $\beta$-versions (beta0 through beta7), while the content of EPICS2017 is released within ENDF/B-VIII.0. The correspondence between $\mathrm{ENDF} / \mathrm{B}$ atomic data libraries and their standalone counterparts is summarized in Table IV.

Physics data are organized according to the ENDF/B hierarchical structure; specific "file" identifiers (MF) correspond to photo-atomic or electro-atomic interaction cross sections $(\mathrm{MF}=23)$, electro-atomic angle and energy distributions $(\mathrm{MF}=26)$, atomic form factors or scattering functions for photo-atomic interactions $(\mathrm{MF}=27)$, and atomic relaxation data $(\mathrm{MF}=28)$.

\section{StRAtegy OF This StUdy}

This paper reports the results of verification and performance tests, which were developed specifically to appraise the impact of using the data libraries released in early 2018 in a Monte Carlo simulation environment.

Verification tests investigated the equivalence of data released in different formats or by different distribution sources, the correspondence of the data with features advertised in the associated documentation, the monotonic character of tabulations required for their correct interpolation and congruity with the newly adopted binding energies. These tests were independent of the use of the data libraries in a specific computational environment.

The evaluation of the performance of the new data libraries addresses the computational resources involved to deal with a major novelty of the 2018 release, i.e. the increase in the number of tabulated data with respect to the previous versions to enable their linear interpolation, while logarithmic interpolation was recommended for the previous data libraries.

Some of the tests to evaluate the performance of the new libraries, namely, those concerning the effect of different interpolation methods, were carried out in a Geant4-based context of use; although their outcome in absolute terms is specific to the implementation of the algorithms and the computational environment where the tests were executed, the relative comparisons reported in Section V are of general interest.

As the outcome of these tests sheds light on the underlying system life-cycle processes, this paper also encompasses some software engineering considerations from the perspective of using the data libraries in a scientific application context.

\section{Assessment of Data Features}

\section{A. Evolution With Respect to the Original Livermore Library}

The data libraries originally released by LLNL are currently widely used by the experimental community, since they are the foundation of electron-photon transport of major Monte Carlo codes. A clear picture of their evolution through EPICS2014 and EPICS2017, the latter embedded in ENDF/ B-VIII, is useful to discern changes that could affect simulation applications.

Although reference [40] states that EPICS2014 has "modernized these data libraries by reviewing recently published data and making changes," the tests performed in this paper identified that the actual content of EPICS2014 is identical to that of the original versions of EADL, EEDL, and EPDL produced by LLNL in 1991 and 1997 currently available in electronic format, with the exception of the correction of format conversion errors mentioned in Section II-B. Therefore, EPICS2017 is the first release that encompasses changes with respect to the original Livermore Library data.

The major change in EPICS2017 concerns the atomic binding energies included in EADL. Although a complete appraisal of this evolution will come from physics validation tests, which are outside the scope of this paper, at this stage one can observe that the sources used to define the binding energies of EPICS2017 were included in the evaluation of [41], where they yielded more reliable results than EADL91 in the various test scenarios examined in that paper.

The effects of modifying atomic binding energies in EADL are propagated into other physics quantities tabulated in EEDL and EPDL. For some physics data sets (e.g. electron impact ionization and photoelectric effect), the same cross section values as in the previous versions are tabulated in EPICS2017 and ENDF/B-VIII.0, but the corresponding interacting particle energies are modified to account for the difference between the old and new electron binding energies.

Apart from electron binding energies, electron kinetic energies also appear to have been modified in EPICS2017 with 


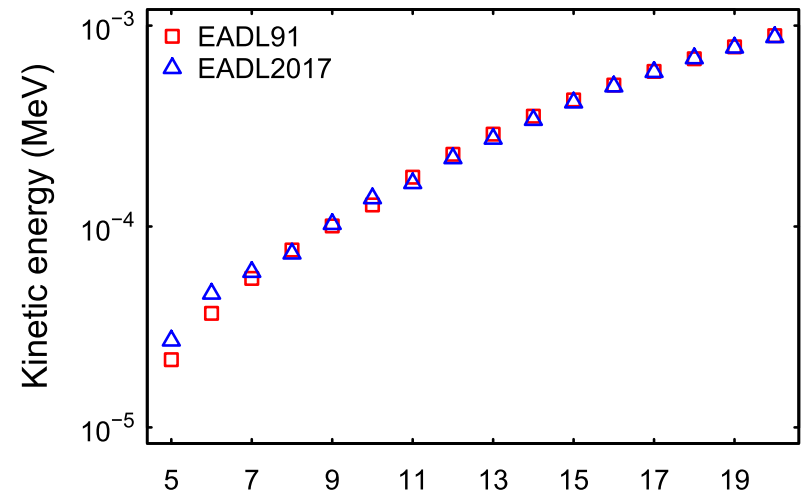

Atomic number $\mathbf{Z}$

Fig. 1. Electron kinetic energy in the $\mathrm{L}_{2}$ subshell as a function of atomic number: in the original 1991 version of EADL released by LLNL (red squares) and in the EADL version included in EPICS2017 (blue triangles). The range of atomic numbers displayed in the figure is limited to highlight the differences mainly concerning light elements.

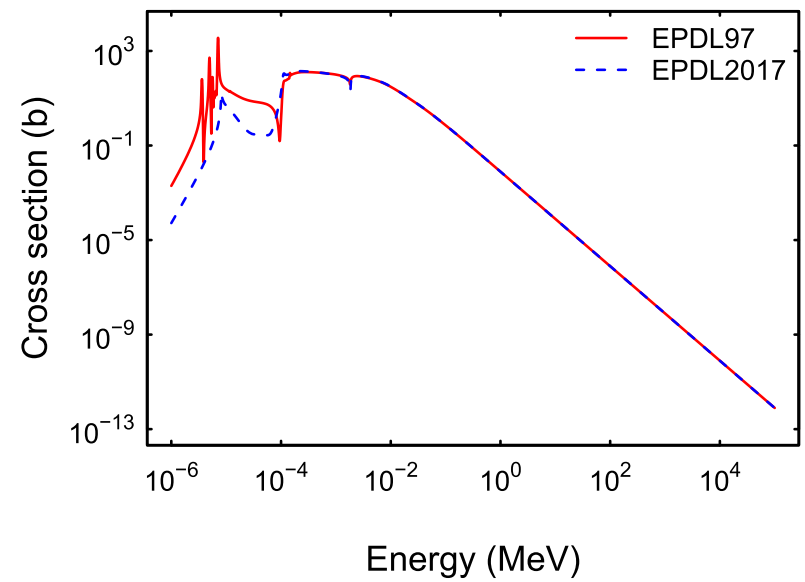

Fig. 2. Integrated cross section of photon coherent scattering with silicon as a function of photon energy.

respect to previous EADL releases. Differences appear larger for elements with low atomic numbers, as one can appreciate in Fig. 1. The origin of the electron kinetic energies included in EADL91 could not be ascertained, as the published references cited in [12] as sources of the data do not mention them; presumably, they pertain to the references indicated in [12] as "private communication." Similarly, [9] does not document the source of the modified electron kinetic energies distributed in EPICS2017.

Large differences are observed in the integrated photon coherent scattering cross sections and in both the real and imaginary components of anomalous scattering factors; an example is illustrated in Fig. 2. Those occurring at very low energies are not relevant to the use of EPDL in Monte Carlo transport codes, as [10] recommends using EPICS2017 for photon transport above $100 \mathrm{eV}$. At higher energies, the scarcity of the experimental measurements of total photon elastic scattering cross sections, reported in [42], makes it impossible to know whether the new total coherent scattering cross sections are more accurate than those included in EPDL97.

A subset of EEDL excitation data (integrated cross sections and average energies), limited to 17 elements, exhibits
TABLE V

RATIO OF THE NuMBER OF DATA POINTS IN EPICS2017 (ENDL FORMAT) AND ENDF/B-VIII.0 WITH RESPECT TO EPDL97

\begin{tabular}{lcc}
\hline \multirow{2}{*}{ Physics data sets } & \multicolumn{2}{c}{ Ratio } \\
& Min & Max \\
\hline Coherent scattering: integrated cross section & 0.25 & 0.998 \\
Coherent scattering: form factor & 6.79 & 13.92 \\
Coherent scattering: imaginary anomalous scattering factor & 0.64 & 1.58 \\
Coherent scattering: real anomalous scattering factor & 0.77 & 2.10 \\
Incoherent scattering: integrated cross section & 3.46 & 3.66 \\
Incoherent scattering: scattering function & 2.61 & 4.23 \\
Photoelectric: integrated cross section & 5.64 & 12.38 \\
Photoelectric: cross section by subshell & 3.79 & 12.38 \\
Pair production: integrated cross section & 2.40 & 3.71 \\
Triplet production: integrated cross section & 2.26 & 2.82 \\
\hline
\end{tabular}

differences between EPICS2017 and the values in the original Livermore Library.

Some data (elastic scattering, large angle scattering, and Bremsstrahlung integrated cross sections) exhibit very small differences between EPICS2017 and previous releases; these discrepancies can be attributed to roundoff errors related to the number of significant digits reported in the tabulations rather than to physical changes.

Another major change in the new data libraries is the increase in the number of tabulated data to enable their linear interpolation instead of logarithmic interpolation, which was recommended in previous versions. According to [10], this change is intended to prevent the mistakes of users who, despite the recommendations documented in previous versions of the data library, interpolated the data linearly, thus compromising the precision of the results.

\section{B. Consistency Issues}

The tests performed in the context of this paper highlighted some intrinsic inconsistencies in the content of the data libraries and discrepancies between the documented and actual features of the data.

According to [10], "starting with EPICS2017 all the data has been linearized [...]. The result is libraries are roughly three (3) times as large (e.g. has about 3 times as many energy points), but it can be accurately interpolated using LIN-LIN interpolation"; nevertheless, not all the photon data in the EPDL component of EPICS2017 and in the photo-atomic library of ENDF/B-VIII.0 are tabulated with a larger number of energy points; actually, in some cases, the number of data points has decreased with respect to EPDL97. This apparent contradiction can be observed in Table V, which reports the ratio between the number of data points with respect to those in EPDL97, for the various categories of photon interaction data; the range of values associated with each physics data set reflects the variation of the number of tabulated data with the atomic number. For some coherent scattering data, the ratio listed in Table $\mathrm{V}$ is smaller than one, which corresponds to fewer data points being tabulated in the new EPDL version with respect to EPDL97.

It is worthwhile to note that the larger number of form factor and scattering function data appearing in Table $\mathrm{V}$ 
only concerns the ENDL format of EPICS2017 released by IAEA, the ENDF-6 format of EPICS2017 released by NNDC and ENDF/B-VIII.0, while the number of data in EPICS2017 released by IAEA in ENDF-6 format is the same as in EPDL97. The difference in the number of EPICS2017 data in the ENDF-6 format released by NNDC and by IAEA is not documented. The observed inconsistency of the size of form factor and scattering function tabulations raises concerns regarding the reliability of simulation results, if data that have not been tabulated with higher granularity are linearly interpolated in conformity with the documentation.

A similar inconsistency also affects the electron interaction data: although a statement concerning the increase in the number of tabulated data in view of their linear interpolation is also reported in [11], the number of electron data tabulated in the EEDL component of EPICS2017 and in the electro-atomic library of ENDF/B-VIII.0 is identical to that in the original EEDL91. Therefore, given that the number of tabulated data is unchanged, logarithmic interpolation, which was recommended for the previous EEDL releases, remains the preferred interpolation method for the electron data in EPICS2017 and ENDF/B-VIII.0.

Form factor and scattering function tabulations in EPICS2017 are also affected by inconsistency in the units. Both the quantities are tabulated as a function of an inverse length $x$, which is defined as $x=\sin (\theta / 2) \lambda$, where $\theta$ is the photon scattering angle and $\lambda$ is its wavelength. In ENDL format [17], $x$ is expressed in $\mathrm{cm}^{-1}$; form factors and scattering functions are tabulated consistently in this format in EPDL97, EPICS 2014, and EPICS2017. The unit of $x$ is $\AA^{-1}$, i.e. $10^{10} \mathrm{~m}^{-1}$ in ENDF-6 format [16]. This unit is adopted in ENDF/B-VIII.0, as well as in the EPDL97 and EPICS2014 versions released in the ENDF-6 format. It is worthwhile to stress that the use of different units in ENDL and ENDF-6 formats is not an inconsistency per se, so long as it is coherent with the documentation of the two formats. In EPICS2017, $x$ is expressed in units of $10^{6} \AA^{-1}$, i.e. $10^{16} \mathrm{~m}^{-1}$, in ENDF-6 format, while the tabulated form factor and scattering function values remain the same as in EPICS2014. This change of units of $x$ is not documented; it is liable to induce errors in Monte Carlo simulations drawing form factors and scattering functions from EPICS2017 in the ENDF format.

Radiative and nonradiative transition energies tabulated in the ENDF-6 format are inconsistent with atomic binding energies, both in ENDF/B-VIII.0 and EPICS2017: the values listed in ENDF/B-VIII.0 atomic relaxation library and in EADL2017 (ENDF-6 format) are the same as in EADL91, i.e. they have not been modified for consistency with the new atomic binding energies adopted in ENDF/B-VIII.0 and EPICS2017. An example is illustrated in Fig. 3, which reports an excerpt of the data for carbon. This inconsistency could result in energy nonconservation, if inconsistent binding energies and transition energies are used in the context of Monte Carlo particle transport. The data tabulated in ENDL format in EPICS2017 are correct.

Inconsistencies are present in the spectra of secondary electrons produced by electron impact ionization, listed in ENDL format in the EEDL component of EPICS 2017. One
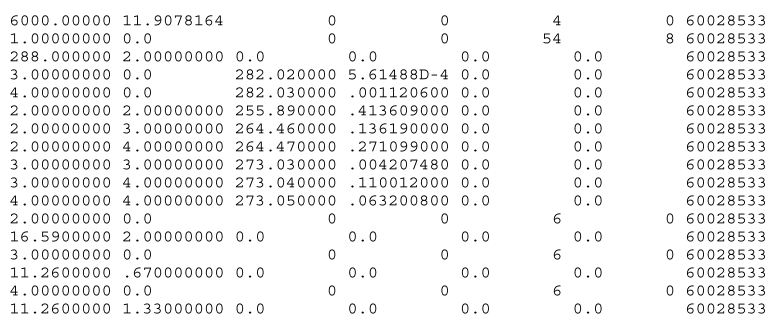

Fig. 3. Inconsistency of atomic relaxation data in ENDF-6 format illustrated in an excerpt of EADL2017 concerning carbon atomic binding energies and transition energies. Radiative transition energies $(282.02$ and $282.03 \mathrm{eV})$ and nonradiative transition energies $(255.89,264.46,264.47,273.03,273.04$, and $273.05 \mathrm{eV}$ ) are inconsistent with the binding energies reported for $\mathrm{K}$ shell $(288.00 \mathrm{eV}), \mathrm{L}_{1}(16.95 \mathrm{eV}), \mathrm{L}_{2}(11.26 \mathrm{eV})$, and $\mathrm{L}_{3}(11.26 \mathrm{eV})$ subshells.

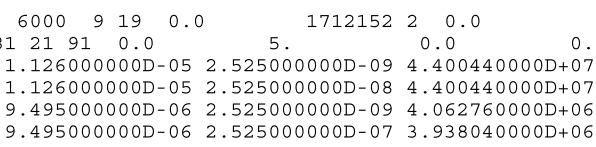

Fig. 4. Inconsistency in the tabulation of secondary electron spectra produced by electron impact ionization in EPICS2017, ENDL format: the EEDL excerpt, concerning carbon ionization, shows primary energies that are not monotonically increasing, as expected for proper interpolation of the data. The tabulation reports secondary electron spectra produced by ionization of the $\mathrm{L}_{2}$ subshell for energies lower than the corresponding electron binding energy $(11.26 \mathrm{eV})$.

can observe cases where tabulated primary electron energies are not monotonically increasing, as is illustrated in Fig. 4: this feature affects proper interpolation of the data. Moreover, as in the excerpt of EEDL reproduced in Fig. 4, the violation of the monotonic ordering of primary electron energies appears associated with energy values lower than the $\mathrm{K}$-shell binding energy of the concerned atom, which is reported in the EADL component of EPICS2017. The data tabulated in ENDF-6 format in EPICS2017 and ENDF/B-VIII.0 are correct.

\section{Physics Issues}

Radiative transition probabilities reported in EADL derive from calculations of transition rates by Scofield [31]-[33], [43]. The procedure to obtain transition probabilities from transition rates, which involves partial and total level widths, is documented in [12].

A previous validation test [44] observed discrepancies between the transition probability values tabulated in EADL and Scofield's original calculations: large differences were visible in $\mathrm{L}_{2}-\mathrm{M}_{1}, \mathrm{~L}_{2}-\mathrm{M}_{4}, \mathrm{~L}_{3}-\mathrm{M}_{1}$, and $\mathrm{L}_{3}-\mathrm{M}_{5}$ transitions. In these cases, radiative transition probabilities directly derived from Scofield's references reproduce the experimental data better than those tabulated in EADL [44]. The same discrepancies are still present in EPICS2017 and ENDF/B-VIII.0.

This issue is illustrated in Figs. 5 and 6, where the probabilities for the $\mathrm{K}-\mathrm{L}_{2}$ and $\mathrm{L}_{2}-\mathrm{M}_{1}$ radiative transitions tabulated in EADL91 and in EPICS2017 (identical to ENDF/B-VIII.0) are compared with those directly derived from Scofield's radiative transition rates listed in [43]. To convert Scofield's transition rates [43] into the transition probabilities plotted in Figs. 5 and 6, the nonradiative level widths tabulated in 


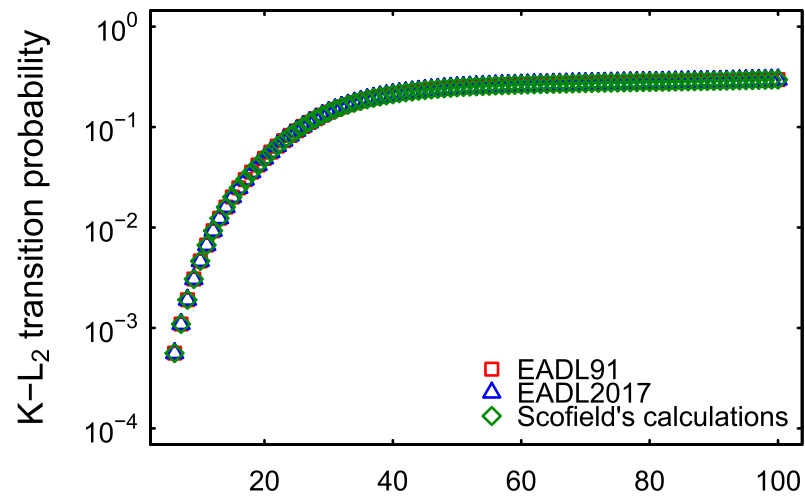

Atomic number $Z$

Fig. 5. $K-L_{2}$ radiative transition probability versus atomic number Z: EADL91 (red squares), EADL2017 (blue triangles) released in EPICS2017 and ENDF/B-VIII.0, and values directly derived from Scofield's calculations [43] (green diamonds).

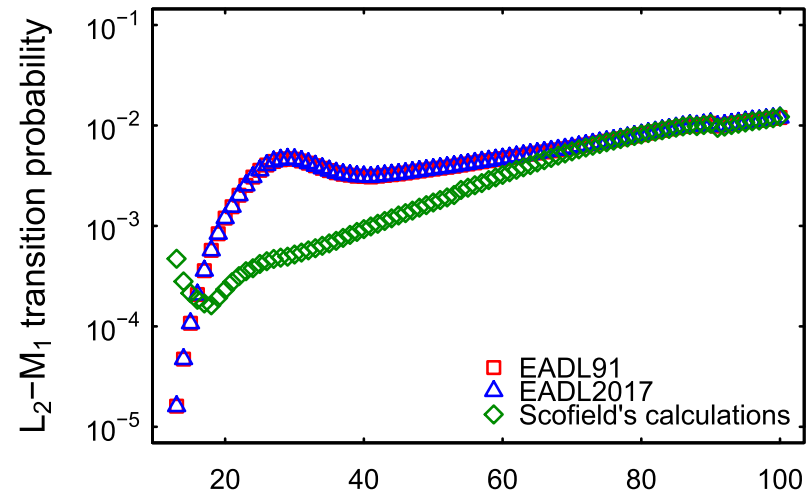

Atomic number Z

Fig. 6. $L_{2}-M_{1}$ radiative transition probability versus atomic number Z: EADL91 (red squares), EADL2017 (blue triangles) released in EPICS2017 and ENDF/B-VIII.0, and values directly derived from Scofield's calculations [43] (green diamonds).

EADL91 in the ENDL format were used. The probabilities for the $\mathrm{K}-\mathrm{L}_{2}$ transition appear to be consistent, while for the $\mathrm{L}_{2}-\mathrm{M}_{1}$ transition, substantial discrepancies are visible with respect to Scofield's [43] original source, especially for lower atomic numbers. It is worthwhile to note that the total radiative level widths reported in EADL are consistent with Scofield's calculations [43], with differences not exceeding $1 \%$.

The results of the validation test [44] identified Scofield's radiative transition probabilities based on the Hartree-Fock calculation method [45], [46] as better reproducing experimental measurements than those based on the Hartree-Slater approach, which are tabulated in the original EADL. Radiative transition probabilities based on the Hartree-Slater calculations are still released in EPICS2017 and ENDF/B-VIII.0.

\section{Reproducibility Issues}

The assessment of EPICS2017 and of the atomic components of ENDF/B-VIII.0 has identified various shortcomings in their configuration management and raised concerns regarding the associated version control.

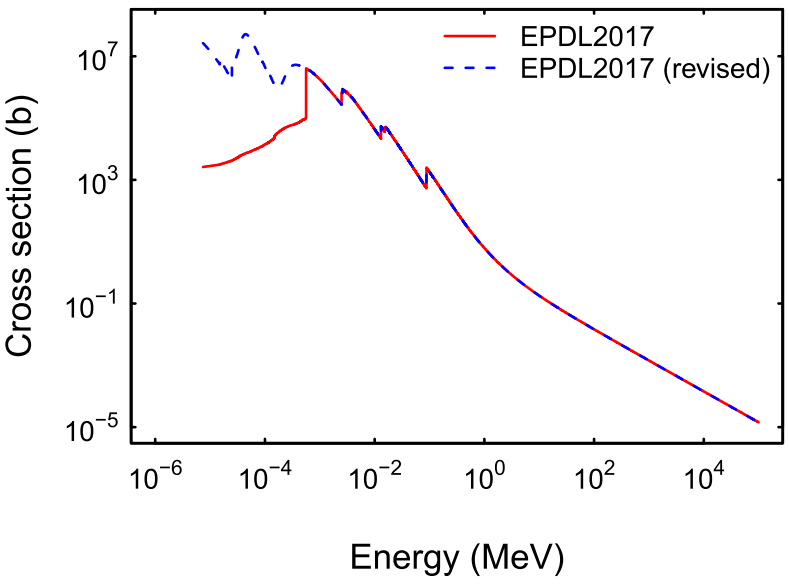

Fig. 7. Integrated photoelectric cross section for lead as a function of photon energy: as in EPICS2017 (red solid line) released by IAEA in January 2018 and in EPICS2017 released by IAEA in February 2018 (blue dashed line).

The collection of different data sets in the data libraries according to the format, ENDF-6 or ENDL, under which they are released, which is documented in Tables I-III, may derive from historical reasons; nevertheless, undocumented inconsistencies exist between the contents of the same library released in both formats, and even between the contents of the same data library released in the same format in different systems, e.g. EPICS2017 and ENDF/B-VIII.0.

Differences are also observed between the data released by IAEA [15] and by NNDC [39] under the same identifier of EPICS2017.

Additionally, different data content has been released by IAEA under the same identifier of EPICS2017. For instance, two versions of the data have been released by IAEA in January 2018 and in February 2018, respectively, encompassing different photoelectric cross sections: an example is illustrated in Fig. 7, which shows the total photoelectric cross sections for lead in the two versions. The occurrence of this change is reported in [10], which is identified as "revision 1," but neither the modified individual library (EPDL) nor the modified collection (EPICS) is tagged as a different version: the two releases are identically identified as EPICS2017 despite their different content.

The lack of proper version control affects the reproducibility of results, which is an essential feature of the scientific method: for instance, it hinders the reproducibility of Monte Carlo simulations which could use different versions of the data, although characterized by the same identifier.

Best practices of configuration management, version control and release management are encoded in standards, such as ISO/IEC/IEEE 12207 [47] and the IEEE Std-828 [48]; they are also documented in textbooks [49], [50] and widely discussed in the literature.

\section{Performance Assessment}

The assessment of the computational performance concerns the evaluation of memory consumption, computational speed, and precision of interpolation associated with the use of 
TABLE VI

Memory Size in Kilobyte Required to USE PHYSICS DATA OF ALL ELEMENTS

\begin{tabular}{|c|c|c|c|}
\hline Physics data & $\begin{array}{l}\text { Original } \\
\text { libraries }\end{array}$ & $\begin{array}{c}\text { ENDF/B-VIII } \\
\text { EPICS2017 }\end{array}$ & Ratio \\
\hline Bremsstrahlung cross section & 368 & 368 & 1 \\
\hline Elastic scattering cross section & 472 & 472 & 1 \\
\hline Large angle elastic scattering cross section & 472 & 472 & 1 \\
\hline Ionisation cross section by subshell & 1924 & 1924 & 1 \\
\hline Excitation cross section & 1152 & 1152 & 1 \\
\hline Coherent scattering cross section & 4528 & 1868 & 0.4 \\
\hline Coherent scattering form factor & 708 & 4620 & 6.5 \\
\hline Incoherent scattering cross section & 508 & 1692 & 3.3 \\
\hline Incoherent scattering scattering function & 724 & 1836 & 2.5 \\
\hline Photoelectric cross section & 4480 & 32620 & 7.3 \\
\hline Photoelectric cross section by subshell & 7536 & 45976 & 6.1 \\
\hline Pair production cross section & 496 & 1356 & 2.7 \\
\hline Triplet production cross section & 436 & 920 & 2.1 \\
\hline
\end{tabular}

the data. The results using the data libraries released in early 2018 are compared with those obtained with the original Livermore libraries.

The performance tests reported in this section relate to the local computational environment where they were executed and, in the case of tests using portions of Geant 4 software, also to Geant4 implementation. Therefore, their results are meaningful as relative indications with respect to using previous versions of the data libraries rather than as absolute requirements of computational resources.

\section{A. Memory Use}

The amount of memory required by the original Livermore libraries and the 2018 version was estimated by loading into memory the whole data content, corresponding to all tabulated elements (with atomic number from $\mathrm{Z}=1$ to 100), for each physics data type. The memory allocated by the Geant 4 objects holding the data was retrieved through the /proc virtual file system, which provides memory information about a process in a Linux operating system environment, by measuring the correspondent VmRSS (Virtual memory Resident Set Size).

The results are summarized in Table VI. This estimate should be considered as a general indication of the relative requirements of the original libraries and those released in early 2018, rather than as absolute estimates of memory consumption. Specific simulation applications may load only portions of the libraries into memory, thus using a smaller amount of resources.

It is worthwhile to note that memory consumption is unchanged for the electron data, since, as discussed in Section IV, the number of electron data tabulated in ENDF/ B-VIII.0 and EPICS2017 is identical to that in the original Livermore libraries, although the documentation [11] states that it has been extended. The reduced memory consumption for coherent scattering integrated cross sections reflects the reduction of this data set, also discussed in Section IV as inconsistent with the documentation [10]. For the other photon interaction data, a substantial increase in memory requirements is observed with respect to the original Livermore libraries.
TABLE VII

Computational Time in Seconds to Calculate Integrated CRoss SECTIONS WITH DiFFERENT INTERPOLATION METHODS

\begin{tabular}{lcc}
\hline Physics process & $\begin{array}{c}\text { Original Libraries } \\
\text { Logarithmic }\end{array}$ & $\begin{array}{c}\text { EPICS2017 } \\
\text { Linear }\end{array}$ \\
\hline Bremsstrahlung & $3.88 \pm 0.01$ & $0.63 \pm 0.01$ \\
Elastic scattering & $3.90 \pm 0.01$ & $0.79 \pm 0.01$ \\
Large angle elastic scattering & $3.92 \pm 0.01$ & $0.79 \pm 0.01$ \\
Excitation & $4.21 \pm 0.01$ & $1.06 \pm 0.01$ \\
Coherent scattering & $4.32 \pm 0.02$ & $1.21 \pm 0.01$ \\
Incoherent scattering & $3.93 \pm 0.01$ & $1.28 \pm 0.01$ \\
Photoelectric & $4.67 \pm 0.01$ & $3.68 \pm 0.01$ \\
Pair production & $2.36 \pm 0.01$ & $0.85 \pm 0.01$ \\
Triplet production & $2.25 \pm 0.01$ & $0.81 \pm 0.01$ \\
\hline
\end{tabular}

\section{B. Computational Speed}

The evaluation of the computational performance associated with the use of the data libraries released in early 2018 was focused on estimating the effect of linear interpolation of the data, consistently with their documentation [10], [11], instead of previously recommended logarithmic interpolation.

Speed tests were executed in a Geant 4 application environment. Thanks to Geant 4 design as a toolkit, only a few objects pertinent to physics data management were instantiated, with minimal dependencies on other parts of the Geant 4 code. The data libraries subject to evaluation were converted into a format suitable to be handled by Geant 4 physics data management classes.

The test scenario for this purpose concerned the calculation of total cross section values using the original Livermore libraries and the libraries released in 2018 along with their pertinent interpolation method. In each test case, $10^{7}$ primary particles were generated with random atomic number uniformly distributed between 1 and 100 and random energies uniformly distributed in logarithmic space between $100 \mathrm{eV}$ and $100 \mathrm{GeV}$. The corresponding cross section values were calculated by linear and logarithmic interpolation of the tabulated data.

The results are summarized in Table VII; they report the time required for the calculation of cross section values, excluding the time for initialization. One can observe that the use of linear interpolation reduces the computational burden substantially with respect to logarithmic interpolation. The gain in computational speed ranges from approximately $30 \%$ for photoelectric cross sections to about a factor 6 for Bremsstrahlung cross sections.

The computational performance results reported here derive from a simple data management software implementation, since the purpose of these tests is to highlight the intrinsic characteristics of the data libraries. Computational performance can be optimized in various ways: for instance, a more efficient algorithm could store precalculated logarithms of the tabulated data in memory to improve the speed of logarithmic interpolation calculations, although at the expense of increased memory consumption; nevertheless, the investigation of data management optimization is beyond the scope of this paper.

\section{Data Interpolation}

According to [10] and [11], the data in EPICS2017 "can be accurately interpolated using LIN-LIN interpolation." 
TABLE VIII

MaXimum Difference Between InTEgRated Cross Sections CALCULATED BY LOGARITHMIC AND LINEAR INTERPOLATIONS: All Data and Data Above $100 \mathrm{eV}$

\begin{tabular}{lrr}
\hline Physics process & $\begin{array}{r}\text { Difference } \\
\text { All data }\end{array}$ & $\begin{array}{r}\text { \% Difference } \\
\mathrm{E}>100 \mathrm{eV}\end{array}$ \\
\hline Large angle elastic scattering & 4.0 & 4.0 \\
Elastic scattering & 1.3 & 1.3 \\
Ionisation & 340.5 & 11.9 \\
Ionisation (by subshell) & 2845.5 & 2845.5 \\
Bremsstrahlung & 1.5 & 1.5 \\
Excitation & 134.5 & 2.1 \\
Coherent scattering & 104.1 & 10.3 \\
Incoherent scattering & 0.1 & 0.1 \\
Photoelectric & 0.1 & 0.1 \\
Photoelectric (by subshell) & 0.1 & 0.1 \\
Pair production & 0.1 & 0.1 \\
Triplet production & 0.1 & 0.1 \\
\hline
\end{tabular}

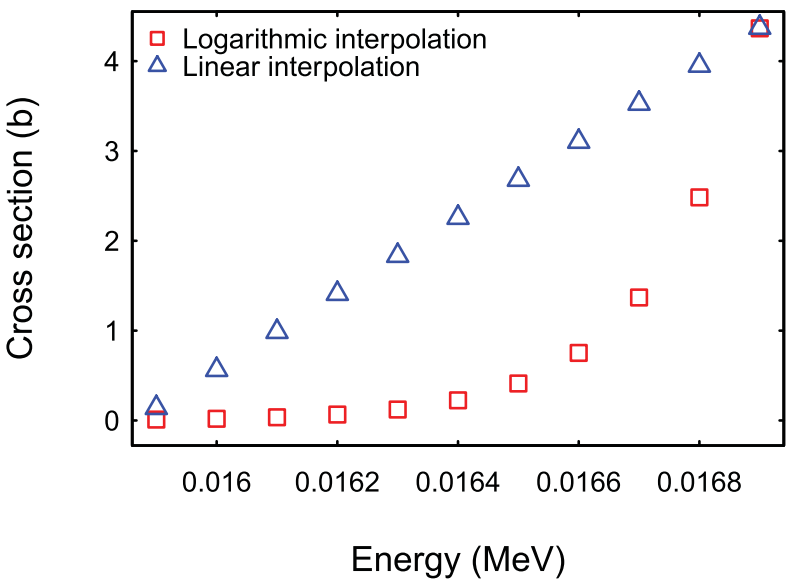

Fig. 8. Cross section for the ionization of the $L_{1}$ shell of lead as a function of electron energy.

The attainable precision was evaluated by estimating the relative difference between the logarithmic and linear interpolations of cross section data. Test cases were configured as described in Section V-B.

The maximum difference between the cross sections calculated by either interpolation method is reported in Table VIII over the whole energy range covered by the data and for energies above $100 \mathrm{eV}$.

No significant difference is observed for photon cross sections with the exception of coherent scattering. It is worthwhile to note that the number of coherent scattering cross section data is reduced in EPICS2017 with respect to the original EPDL97 content; presumably, the smaller number of data affects the precision of their linear interpolation.

Large differences are observed for some electron interaction cross sections; an example is illustrated in Fig. 8. For these data, it is questionable whether the precision achieved by linear interpolation would be adequate for critical Monte Carlo simulation scenarios.

Since the number of data tabulated for electron processes is unchanged with respect to the original EEDL91, which is normally used along with logarithmic interpolation algorithms in Monte Carlo transport codes, users may want to adhere to

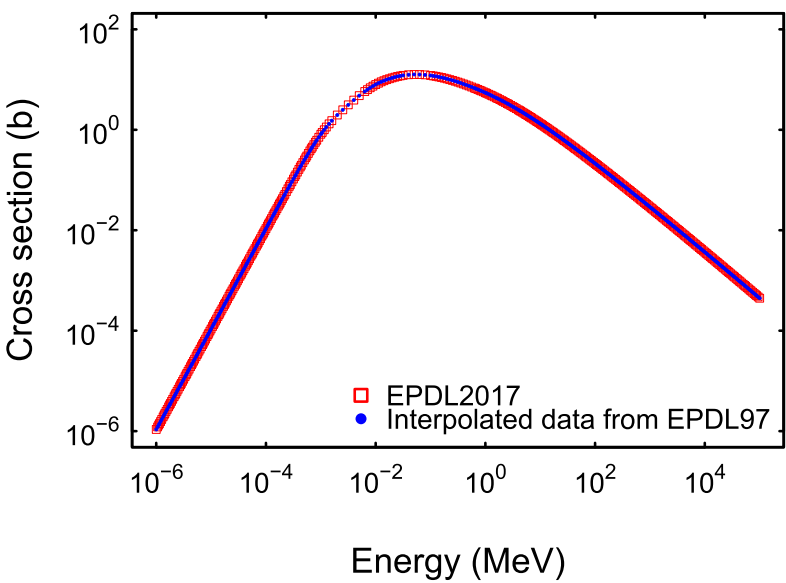

Fig. 9. Integrated cross section for the incoherent scattering of photons with iron as a function of photon energy.

the current practice when using the EEDL2017 data despite the claim of accuracy of linear interpolation in [11].

A practically interesting finding of this assessment is that, for physics data sets not affected by the change of atomic binding energies, the additional data points tabulated in EPDL2017 can be obtained by the logarithmic interpolation of the original EPDL97 with precision better than $0.01 \%$. An example is illustrated in Fig. 9, where cross sections for incoherent scattering of photons with iron tabulated in EPICS2017 and interpolated from EPDL97 are compared: the two data distributions are indistinguishable. The hypothesis of their equivalence fails to be rejected by the KolmogorovSmirnov goodness-of-fit test with 0.01 significance. This finding hints at the possibility for library users to produce tabulations with optimized granularity for their experimental application scenarios based on the original Livermore Library data.

Although the issue of data interpolation is discussed in this section in terms of EPICS2017, the assessment holds for ENDF/B-VIII.0 data as well.

\section{CONCLUSION}

The release of new evaluated atomic data libraries, intended to replace those dating back to the 1990s currently used by major Monte Carlo codes, is a major event in the field, since these libraries are the foundation of electron and photon interaction modeling in widely used simulation systems.

The modification of the atomic binding energies previously included in EADL91, which were identified [41] as a source of inaccuracy and of systematic effects, is a significant feature of EPICS2017 and ENDF/B-VIII.0. Since the sources of the binding energies included in the new EADL helped produce more accurate physics observables than EADL91 in the evaluation of [41], the evolution of EPICS2017 and ENDF/B-VIII.0 looks promising, although its effects should be confirmed by validation tests, i.e. comparisons with experimental measurements of related physics observables, which are outside the scope of this paper.

Nevertheless, the propagation of modified binding energies to the calculation of dependent quantities tabulated in the 
data libraries raises some issues: inconsistencies are observed, which are liable to cause disruptive effects in Monte Carlo simulations. They suggest shortcomings in the change management process adopted in the development of EPICS2017, particularly in the traceability process, and in the test process of both EPICS2017 and ENDF/B-VIII.0 prior to their public release.

A major evolution announced in the documentation, consisting of the extension of the number of tabulated data to enable their linear interpolation, is only partially enacted in the new data libraries. This change, whose motivations do not appear to derive from physics requirements [10], is expected to generate a significant burden on many users of the data libraries, who will be obliged to modify their data management software accordingly. The inconsistency between the data and their documentation risks generating confusion in the user community and unreliable physics results: users who interpolate data linearly in conformity to the documentation [13] are liable to degrade the accuracy of their simulations if the granularity of the tabulations is actually inadequate for this use.

The move from logarithmic to linear interpolation of the data has controversial effects on the computational resources needed to use the libraries, being a tradeoff between memory requirements and computational speed. The results documented in this assessment provide quantitative indications regarding these aspects in an application environment, which the users can adapt to their own experimental scenarios.

The presence of inconsistencies in the data libraries, in any format and source of distribution, complicates their use in an experimental application environment. Although this assessment provides some guidelines by identifying the shortcomings of the data sets that have to be addressed in a simulation production environment, users are responsible for applying the necessary corrective actions to ensure the reliability of their results. It is desirable that the responsible distribution centers consider releasing a corrected version of the data libraries, incorporating solutions to the problems pointed up in this paper, as its availabilty would significantly improve the ease and the dependability of use in experimental scenarios.

A major source of concern emerging from this assessment is the lack of proper version control in the release process: multiple versions of the data exist, with inconsistent content between the different formats in which they are available and the different distribution sources from which they originate. Additionally, a case was observed where the same distribution source released a modified version of the data under the same release identifier (EPICS2017). The lack of proper version control of the data affects the configuration management in the experimental applications that use them, generating confusion about the conditions in which physics results are produced and affecting their reproducibility. It is warmly recommended that the developers and the distribution sites of these data libraries adopt a common and unique protocol for their configuration management and version management, so that the conditions of use of the data libraries can be univocally identified.

\section{ACKNOWLEDGMENT}

The authors would like to thank D. E. Cullen for valuable information about the production process of the EPICS2017 data library and associated reports. The CERN Library has provided helpful assistance and essential reference material for this paper. They would also like to thank A. Hollier for proofreading this paper. E. Salvo and the Computing Service of INFN Genova provided critical support to the computing infrastructure used for this assessment.

\section{REFERENCES}

[1] A. V. Ignatyuk and B. I. Fursov, "The latest BROND-3 developments," in Proc. Int. Conf. Nucl. Data Sci. Technol., 2008, pp. 759-763.

[2] Z. G. Ge, Z. X. Zhao, and H. H. Xia, "The updated version of chinese evaluted nuclear data library (CENDL-3.1)," J. Korean Phys. Soc., vol. 59, no. 2, pp. 1052-1056, 2011.

[3] D. A. Brown et al., "ENDF/B-VIII.0: The $8^{\text {th }}$ major release of the nuclear reaction data library with CIELO-project cross sections, new standards and thermal scattering data," Nucl. Data Sheets, vol. 148, pp. 1-142, Feb. 2018

[4] A. J. Koning et al., "Status of the JEFF nuclear data library," J. Korean Phys. Soc., vol. 59, no. 2, pp. 1057-1062, 2011.

[5] K. Shibata, O. Iwamoto, T. Nakagawa, N. Iwamoto, A. Ichihara, and S. Kunieda, "JENDL-4.0: A new library for nuclear science and engineering," J. Nucl. Sci. Technol., vol. 48, no. 1, pp. 1-30, 2011.

[6] C. L. Dunford, "Evaluated nuclear data file (ENDF)," in Nuclear Data for Science and Technology. Berlin, Germany: Springer, 1992, pp. 788-792.

[7] M. B. Chadwick et al., "ENDF/B-VII.0: Next generation evaluated nuclear data library for nuclear science and technology," Nucl. Data Sheets, vol. 107, pp. 2931-3060, 2011.

[8] IEEE Standard for System, Software, and Hardware Verification and Validation, IEEE Standard 1012-2016, 2017.

[9] D. E. Cullen, A Survey of Atomic Binding Energies for Use in EPICS2017, document IAEA-NDS-0224, Vienna, Austria, 2017.

[10] D. E. Cullen, A Survey of Photon Cross Section Data for Use in EPICS2017, Rev. 1, document IAEA-NDS-0225, Vienna, Austria, 2017.

[11] D. E. Cullen, A Survey of Electron Cross Section Data for use in EPICS2017, document IAEA-NDS-0226, Vienna, Austria, 2017.

[12] S. T. Perkins, M. H. Chen, D. E. Cullen, and J. H. Hubbell, "Tables and graphs of atomic subshell and relaxation data derived from the LLNL evaluated atomic data library (EADL), Z=1-100," Lawrence Livermore Nat. Lab., Tech. Rep. UCRL-50400, Livermore, CA, USA, 1991, vol. 30.

[13] S. T. Perkins, D. E. Cullen, and S. M. Seltzer, "Tables and graphs of electron-interaction cross sections from $10 \mathrm{ev}$ to $100 \mathrm{GeV}$ Derived from the LLNL evaluated electron data library (EEDL)," Lawrence Livermore Nat. Lab., Livermore, CA, USA, Tech. Rep. UCRL-50400, 1991, vol. 31 .

[14] D. E. Cullen, J. H. Hubbell, and L. Kissel, "EPDL97: The evaluated photon data library, rev. 5," Lawrence Livermore Nat. Lab., Livermore, CA, USA, Tech. Rep. UCRL-50400, 1997, vol. 6.

[15] EPICS. IAEA Distribution. [Online]. Available: https://wwwnds.iaea.org/epics/

[16] A. Trkov, M. Herman, and D. A. Brown, "ENDF-6 formats manual," Brookhaven Nat. Lab., Upton, NY, USA, Tech. Rep. BNL-203218-2018INRE, 2018.

[17] S. T. Perkins and D. E. Cullen, "ENDL type formats for the LLNL evaluated atomic data library (EADL), evaluated electron data library (EEDL), and evaluated photon data library (EPDL), rev. 1," Lawrence Livermore Nat. Lab., Tech. Rep. UCRL-ID-117796, 2002.

[18] I. Kawrakow, E. Mainegra-Hing, D. W. O. Rogers, F. Tessier, and B. R. B. Walters, "The EGSnrc code system: Monte Carlo simulation of electron and photon transport," Nat. Res. Council Canada, Ottawa, ON, Canada, Tech. Rep. NRCC PIRS-701, 2017.

[19] T. T. Böhlen et al., "The FLUKA code: Developments and challenges for high energy and medical applications," Nucl. Data Sheets, vol. 120, pp. 211-214, Apr. 2014.

[20] A. Ferrari, P. R. Sala, A. Fasso, and J. Ranft, "FLUKA: A multiparticle transport code," Geneva, Switzerland, Tech. Rep. CERN-2005010, INFN/TC-05/11, SLAC-R-773, Oct. 2005.

[21] S. Agostinelli et al., "Geant4-A simulation toolkit," Nucl. Instrum Methods Phys. Res. A, Accel. Spectrom. Detect. Assoc. Equip., vol. 506, no. 3, pp. 250-303, 2003 . 
[22] J. Allison et al., "Geant4 developments and applications," IEEE Trans. Nucl. Sci., vol. 53, no. 1, pp. 270-278, Feb. 2006.

[23] J. Allison et al., "Recent developments in Geant4," Nucl. Instrum. Methods Phys. Res. Section A, Accel., Spectrometers, Detect. Assoc. Equip., vol. 835, pp. 186-225, Nov. 2016.

[24] T. Goorley et al., "Initial MCNP6 release overview," Nucl. Technol., vol. 180, no. 3, pp. 298-315, 2012.

[25] F. Salvat, J. M. Fernández-Varea, and J. Sempau, "Penelope-A code system for Monte Carlo simulation of electron and photon transport," Boulogne-Billancourt, France, Tech. Rep. NEA/NSC/DOC(2015)3, 2015.

[26] E. F. Plechaty and J. R. Terrall, "Photon cross sections $1 \mathrm{keV}$ to 100 MeV," Lawrence Radiat. Lab., Berkeley, CA, USA, Tech. Rep. UCRL-50400, 1968, vol. 6

[27] E. F. Plechaty, D. E. Cullen, and R. J. Howerton, "Tables and graphs of photon-interaction cross sections from $0.1 \mathrm{keV}$ to $100 \mathrm{MeV}$ derived from the LLL evaluated-nuclear-data library, rev. 1," Lawrence Livermore Lab., Livermore, CA, USA, Tech. Rep. UCRL-50400, 1975, vol. 6.

[28] E. F. Plechaty, D. E. Cullen, and R. J. Howerton, "Tables and graphs of photon-interaction cross sections from $0.1 \mathrm{keV}$ to $100 \mathrm{MeV}$ derived from the LLL evaluated-nuclear-data library, rev. 2," Lawrence Livermore Lab., Livermore, CA, USA, Tech. Rep. UCRL-50400, 1978, vol. 6.

[29] E. F. Plechaty, D. E. Cullen, and R. J. Howerton, "Tables and graphs of photon-interaction cross sections from $0.1 \mathrm{keV}$ to $100 \mathrm{MeV}$ derived from the LLL evaluated-nuclear-data library, rev. 3," Lawrence Livermore Lab., Livermore, CA, USA, Tech. Rep. UCRL-50400, 1981, vol. 6.

[30] D. E. Cullen et al., "Tables and graphs of photon interaction cross sections from $10 \mathrm{eV}$ to $100 \mathrm{GeV}$ derived from the LLNL evaluated photon data library (EPDL), rev. 4," Lawrence Livermore Lab., Livermore, CA, USA, Tech. Rep. UCRL-50400, 1989, vol. 6.

[31] J. H. Scofield, "Radiative decay rates of vacancies in the K and L shells," Phys. Rev., vol. 179, no. 1, pp. 9-16, 1969.

[32] J. H. Scofield, "Relativistic Hartree-Slater values for $K$ and $L$ $X$-ray emission rates," Atom. Data Nucl. Data Tables, vol. 14, no. 2, pp. 121-137, 1974.

[33] J. H. Scofield, "Radiative transitions," in Atomic Inner Shell Processes, vol. 6, B. Craseman, Ed. New York, NY, USA: Academic, 1975.

[34] M. H. Chen, B. Craseman, and H. Mark, "Relativistic radiationless transition probabilities for atomic K- and L-shells," At. Data Nucl. Data Tables, vol. 24, no. 1, pp. 13-37, 1979.

[35] M. H. Chen, B. Craseman, and H. Mark, "Relativistic $K$-shell Auger rates, level widths and fluorescence yields," Phys. Rev. A, vol. 21, no. 2, pp. 436-449, 1980.
[36] M. H. Chen, B. Craseman, and H. Mark, "Relativistic M-shell radiationless transitions," Phys. Rev. A, vol. 21, no. 2, pp. 449-453, 1980.

[37] M. H. Chen, B. Craseman, and H. Mark, "Widths and fluorescence yields of atomic L-shell vacancy states," Phys. Rev. A, vol. 24, no. 1, pp. 177-182, 1981.

[38] S. M. Seltzer, "Cross sections for bremsstrahlung production and electron-impact ionization," in Monte Carlo Transport of Electrons and Photons, T. M. Jenkins and W. R. Nelson, Ed. New York, NY, USA: Plenum Press, 1988.

[39] EPICS. NNDC Distribution. [Online]. Available: https://www.nndc.-bnl.gov/-endf/-epics/

[40] D. E. Cullen, "EPICS2014: Electron photon interaction cross sections (version 2014), rev. 1," Vienna, Austria, Tech. Rep. IAEA-NDS-218, 2015.

[41] M. G. Pia et al., "Evaluation of atomic electron binding energies for Monte Carlo particle transport," IEEE Trans. Nucl. Sci., vol. 58, no. 6 , pp. 3246-3268, Dec. 2011.

[42] M. Batic, G. Hoff, M. G. Pia, and P. Saracco, "Photon elastic scattering simulation: Validation and improvements to geant4," IEEE Trans. Nucl. Sci., vol. 59, no. 4, pp. 1636-1664, Aug. 2012.

[43] J. H. Scofield, "Theoretical radiative transition rates for K- and L-shell X rays," Lawrence Livermore Lab., Livermore, CA, USA, Tech. Rep. UCRL-51231, 1972.

[44] M. G. Pia, P. Saracco, and M. Sudhakar, "Validation of K and L shell radiative transition probability calculations," IEEE Trans. Nucl. Sci., vol. 56, no. 6, pp. 3650-3661, Dec. 2009

[45] J. H. Scofield, "Exchange corrections of K X-ray emission rates," Phys. Rev. A, vol. 9, no. 2, pp. 1041-1049, 1974.

[46] J. H. Scofield, "Hartree-Fock values of L X-ray emission rates," Phys. Rev. A, vol. 10, no. 5, pp. 1507-1510, 1974

[47] Systems and Software Engineering-Software Life Cycle Processes, ISO/IEC/IEEE 12207:2017, Geneva, Switzerland, 2017.

[48] IEEE Standard for Configuration Management in Systems and Software Engineering, IEEE Standard 828-2012, IEEE Computer Society, 2012, pp. 1-71.

[49] I. Sommerville, "Configuration management," in Software Engineering, 10th ed. Boston, MA, USA: Pearson, 2015, ch. 25, pp. $730-755$.

[50] B. Aiello and L. A. Sachs, Configuration Management Best Practices: Practical Methods That Work in the Real World. Boston, MA, USA: Pearson, 2010. 